\title{
Creating Single-Subject Research Design Graphs with Google Applications
}

\author{
Bryan J. Blair $^{1}$ (I) $\cdot$ Paul J. Mahoney ${ }^{2}$
}

Accepted: 26 May 2021 / Published online: 29 November 2021

(C) Association for Behavior Analysis International 2021

\begin{abstract}
The visual analysis of graphically displayed data is an essential skill for behavior analysts across a range of settings. Although there are several computer-based applications that facilitate the production of simple, consistent, and visually coherent graphs, these applications have several limitations, including cost. An alternative to using these applications is using free and widely available Google Sheets and Google Slides to produce high-quality clinical and research graphs. We provide a step-by-step pictorially supported task analysis for a system for creating graphs for a variety of single-subject research designs and clinical applications using Sheets and Slides. We also discuss the advantages and limitations of using Google applications to create graphs for use in the practice of applied behavior analysis.
\end{abstract}

Keywords line graph $\cdot$ Google Sheets $\cdot$ Google Slides $\cdot$ single-subject research design $\cdot$ technology

One of the defining characteristics of applied behavior analysis (ABA) is maintaining direct contact with program data to facilitate appropriate and effective clinical and research decision making. To maintain such contact with data, several computer applications have been used by researchers and practitioners. However, many of these applications are limited by cost, accessibility, customization flexibility, and the fact that in general they restrict users to local usage. Applications like Microsoft Excel, GraphPad Prism, Apple Numbers, and Systat SigmaPlot are used by behavior analysts, however, given our experiences, Google applications have not enjoyed such widespread usage. Google offers access to several cloud-based productivity applications, storage options, and interactive capabilities that permit users to collaborate in real-time. Google Sheets, a spreadsheet application similar to Excel and Numbers, provides a platform in which ABA researchers and practitioners may summarize, analyze, and share applicable data in real time. For the ABA service provider, where cost may be a concern that drives which means of data collection and analysis is employed, Google offers several

Bryan J. Blair

bryan.blair@liu.edu

1 Long Island University-Brooklyn, 1 University Plaza, Brooklyn, NY 11201, USA

2 Amego, Inc., Attleboro, MA, USA applications and features free of charge; however, paymentbased subscriptions are available depending on security and storage needs (e.g., when managing protected health information).

Although some agencies have recently begun to use software applications that automatically summarize clinical data to produce graphs, in general the graphs that are automatically generated are not suitable for professional publication and thus the raw data must still be exported and manipulated in applications like Excel, Prism, Numbers, and now even Sheets. Excel, Prism, Numbers, SigmaPlot, and Sheets all include features that allow users to graph clinical data for manuscript submission or for conference presentations in ways that many other applications do not.

Several technical articles for the development of singlesubject research design graphs have been published to assist ABA practitioners and researchers in their usage. For example, Carr and Burkholder (1998), Dixon et al. (2009), and Pritchard (2008) published tutorials on generating graphs with Microsoft Excel whereas Berkman et al. (2018) published a tutorial on training staff to create graphs using GraphPad Prism. Vanselow and Bourret (2012) also provided the ABA community with online interactive tutorials for creating a variety of graphs in Excel. In addition, other authors have published pictorially supported task-analyzed technical tutorials for using free/low-cost web-based collaborative applications to develop behavior analytic instructional activities (Blair \& Shawler, 2019; Mattson et al., 2020). Likewise, the purpose of this technical article is to provide 
a tutorial specific to generating graphs common to ABA practice and research using web-based Google applications.

\section{Why Google Sheets and Slides? Why Now?}

Data analysis and graphing applications like Excel, Prism, Numbers, and SigmaPlot are fully featured applications that are well-suited to the needs of behavior analysts. But all of these applications present users with specific limitations (see Table 1). For example, the only native application that is free is Numbers, but only for macOS users. Another limitation is where users can use the applications, for example, SigmaPlot can only be used on the Windows operating system (OS). Although Excel and Prism can be used on both macOS and Windows OS, Prism cannot be used on Chrome OS and Excel can only be used with many limitations on Chrome OS through the Excel web application. Numbers can also be used as a web application on macOS, Windows OS, and Chrome OS, but, like Excel, the web application is missing some critical editing features when compared to its native application. A final limitation is the lack of ability to view graphs across operating systems without exporting or converting to a PDF, JPG, or GIF file. Prism and SigmaPlot are fully featured and comprehensive graph production applications, but they are both limited because graphs must first be converted/exported before being shared with others who might not have access to those applications.

The newest version of Sheets addresses all of these specific limitations. When compared to other data summary, data analysis, and graph production applications, Sheets presents several advantages, including the fact that it is free in almost all general use cases, it can be used on nearly any computer, graphs can be easily viewed on nearly any device, it can be used on mobile devices, and it allows for synchronous collaboration across users. In addition, when using a graph development and presentation system with Sheets and Slides, clinicians and researchers can present high-quality, clear, illustrative, minimalistic, and visually coherent graphs across a range of settings and presentation modalities (e.g., in-person, synchronous video meeting, asynchronous sharing). In particular, Google applications allow users to link data and graphs from within Sheets to Slides so that any changes made within Sheets are automatically updated within Slides as well.

These collaborative and synchronization features allow clinicians and researchers to be confident that all members of a team have access to the most recent data and the most recent visual presentation of that data. In contrast to more static applications with collaborative editing and visual consistency limitations like Excel, Prism, Numbers, or SigmaPlot, in particular during times when remote collaboration is even more critical, the dynamically linked and visually consistent Sheets

Table 1 Preferred Characteristics of Single-Subject Research Design Graphs and Features of Common Graph Production Applications

Google Sheets Google Sheets Microsoft GraphPad Systat Apple

(Prior to Update) (After Update) Excel Prism SigmaPlot Numbers

Preferred Formatting Characteristic

1. Tick marks and labels

2. Tick marks outside of axis lines

3. No gridlines or minor tick marks

4. Aligned tick mark, tick mark label, and data point

5. Thin data series lines and large data markers

6. Disconnected data series lines across phases/ conditions

7. Phase/condition lines fall between tick marks

8 . Force both axis lines to appear on graph

9. First session not on vertical (y) axis

10. Floating zero

Usability Characteristic

Cost

All formatting features available on free web-based version of application

All formatting features available on iPadOS application

Graphs viewable in native file format or via web browser on several operating systems (macOS, Windows OS, Chrome OS, iPadOS, iOS $)^{2}$

\begin{tabular}{|c|c|c|c|c|c|}
\hline No & Yes & Yes & Yes & Yes & Yes \\
\hline No & Yes & Yes & Yes & Yes & Yes \\
\hline Yes & Yes & Yes & Yes & Yes & Yes \\
\hline No & Yes & Yes & Yes & Yes & Yes \\
\hline Yes & Yes & Yes & Yes & Yes & Yes \\
\hline Yes & Yes & Yes & Yes & Yes & Yes \\
\hline No & Yes $^{1}$ & Yes & Yes & Yes & Yes \\
\hline $\begin{array}{l}\text { Yes (but } \\
\quad \text { inconsistent) }\end{array}$ & Yes & Yes & Yes & Yes & Yes \\
\hline Yes & Yes & Yes & Yes & Yes & Yes \\
\hline No & Yes & Yes & Yes & Yes & Yes \\
\hline Free & Free & $\$ 69.99 / \mathrm{yr}$ & $\$ 276 / \mathrm{yr}$ & $\$ 899$ & Free \\
\hline No & Yes & No & N/A & N/A & No \\
\hline No & No & No & N/A & N/A & No \\
\hline Yes & Yes & Yes & No & No & Yes \\
\hline
\end{tabular}

\footnotetext{
${ }^{1}$ Accomplished with Slides.

${ }^{2}$ Some formatting inconsistencies are possible.
} 
and Slides system can be advantageous across a range of practices. The COVID-19 pandemic has possibly accelerated this need for remote collaboration. The pandemic imposed several restrictions that have significantly impacted traditional behavior analytic research and practice, and these restrictions have resulted in an increased demand for remote-based services and have altered the way in which behavior analysts conduct their research and clinical practice.

Despite the advantages of using Sheets and Slides related to collaboration and data synchronization, many ABA practitioners may not have previously used Sheets given a history of several design and customized formatting limitations when compared to other applications. However, on June 29, 2020, Google updated Sheets to allow for more precise design and formatting options that are relevant to single-subject research design graphs (Cooper et al., 2020; Kubina et al., 2017) and clinical data presentations (Google LLC, 2020). In particular, users can now add tick marks and tick mark labels, floating zeros, and visible axis lines to their graphs. In addition, users can now align data markers with tick marks. Table 1 lists several critical features added to the Sheets application with the update in June. Figure 1 provides examples of several new features included in the most recent version of the application. Many BCBAs might use Google applications in their clinical and/or research practice; however, they might not be aware of these new capabilities in Sheets. We believe that these updates make Sheets much more suitable for use in many ABA settings and provide a viable alternative to paid applications and/or standalone applications that can only be used effectively and consistently with specific operating systems.

The following pictorially supported step-by-step task analysis provides ABA practitioners and researchers with easy-tofollow instructions to create and collaboratively maintain, in real time, commonly used single-subject research design line graphs that can also be automatically updated for access across colleagues, parents, caretakers, and other stakeholders across geographical locations. This tutorial allows for the creation of simple common line graphs but is not intended for graphs with detailed formatting requirements (e.g., editing individual tick mark labels, creating axis breaks, implementing discontinuous axis values, complex legends). These instructions result in graphs that are high-quality, visually consistent, and suitable for the majority of clinical settings with a quality that rivals graphs published in widely read behavior-analytic journals.

The tutorial begins with instructions for how to create a simple multiple condition/phase (e.g., withdrawal research design) line graph. The general steps for the development of the line graphs are as follows:

1. Create the data table in Sheets;

2. Create the graph from the data in Sheets;

3. Finalize formatting of the graph in Sheets;

4. Make a copy of the graph in Sheets to reuse formatted graph for future graphs;

5. Copy and link graph to Slides;

6. Perform final editing of graphs in Slides.

These basic steps can then be used to create multielement (alternating treatments) and multiple baseline research design graphs because the basic formatting is the same for each. Tiered multiple baseline research design graphs can be created by making multiple A-B graphs and then using Slides for alignment and formatting, multielement research design graphs can be created by using multiple columns of data in the data table and by making formatting changes in Sheets and Slides (e.g., adding a legend and changing data point shapes), and changing criterion research design graphs can be created by adding specific formatting (e.g., criterion lines) in Slides.
Fig. 1 An Example of a Graph with the Identified Preferred Characteristics (View Together with Table 1). Note: This figure is an illustration of recommended preferred characteristics of a single-subject research design graph. The numbers correspond with the written descriptions of the preferred characteristics in Table 1

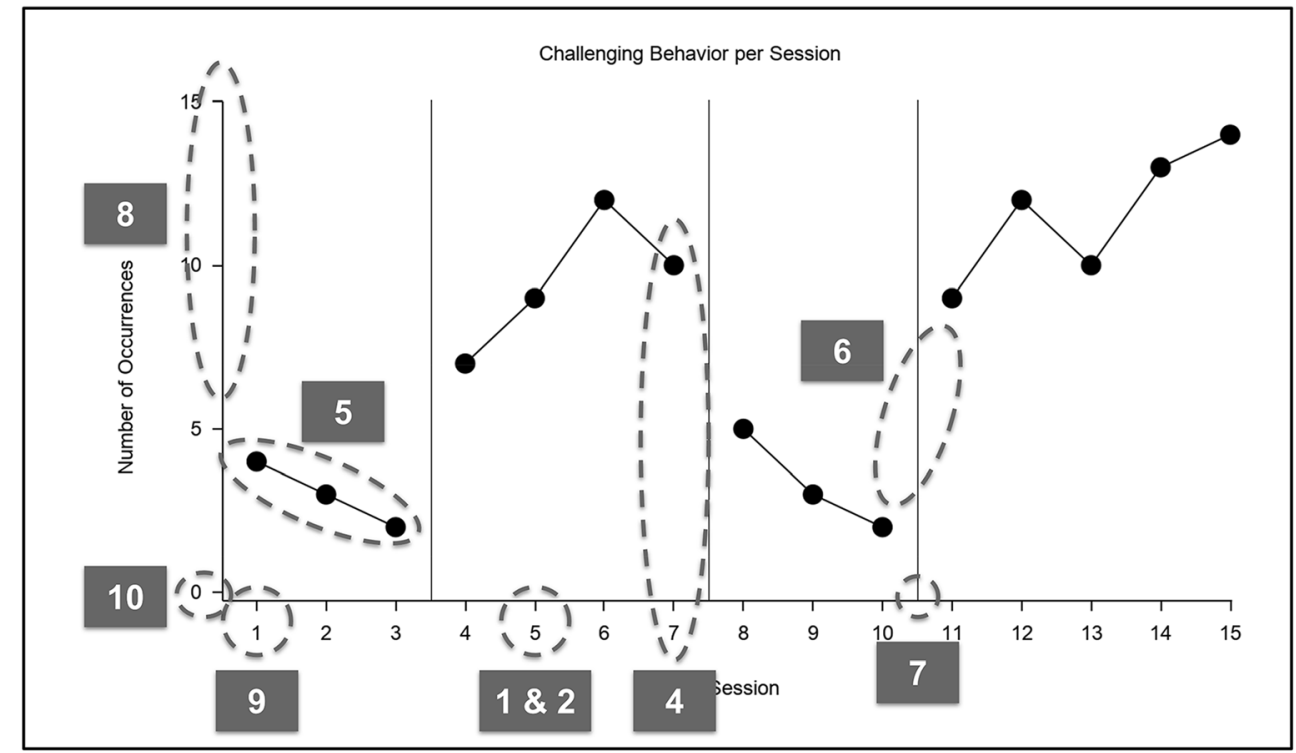




\section{Some General Comments and Observations on the Behavior of Google Sheets}

\section{Regarding Template Graphs}

Although we do recommend creating template graphs in Sheets and then duplicating tabs within an individual spreadsheet, or duplicating an entire Sheets file for future graphs, we also suggest that if Sheets does not behave as expected (i.e., given a large number of possible permutations and combinations of connected settings and formatting options), it is probably easier and quicker to simply create a new blank Sheets file/tab and to follow the taskanalyzed steps below.

\section{Regarding Data Series Line Connectedness and Plotting Null Values}

Given that Sheets does not have a feature for formatting individual data series lines, if the graph requires connected data paths even when data do not appear in subsequent rows in a data table (e.g., multielement design graphs) select Plot null values in the Chart style menu. Plotting null values (i.e., connecting data across periods of time of no data collection) can also be considered in order to skip data for particular sessions (e.g., multiple probe research design) or dates (e.g., weekends) whereas the session number/date and tick marks appear on the horizontal (x) axis. To keep lines disconnected even when null values are plotted (e.g., skipped dates in an ABAB withdrawal research design), instead of inputting data from the same condition in the same column after a gap in data collection for a certain condition (e.g., when switching between baseline and intervention multiple times), new columns with consistent data series names should be created in the data table for every condition/ phase change, and data marker shapes must be changed to ensure conformity within conditions. Provided these considerations, the following sections outline easy-tofollow steps to construct commonly used graphs ABA research and practice.

\section{Creating a Simple Withdrawal Design Graph}

Data Table and Graph Insert (Fig. 2)

1. Create a data table in Sheets with session/date, baseline, and treatment/intervention column headers (or other applicable data series/condition name);

2. Enter data by condition and by session number or date;

3. Highlight all rows and columns;
4. Click Insert $>$ Chart - (it should default to Line chart but if it doesn't, change chart type to Line chart in the Chart editor to the right $)^{1}$;

General Formatting (Figs. 3, 4, 5 and 6)

1. Double-click on legend and press delete on keyboard $^{2}$;

2. Double-click on graph title and press delete on keyboard;

3. Double-click on vertical (y) axis on $\operatorname{graph}^{3}$ (Fig. 3); 1. Click on Gridlines and ticks;

1. Deselect Major gridlines;

2. Select Major ticks;

3. Set Ticks position to outside;

4. Set Ticks length to $\mathbf{6}$;

5. Set Line color to black.

4. Repeat step 3 for horizontal (x) axis.

5. Double-click on vertical (x) axis on graph (Fig. 4);

1. Select Show axis line (if applicable);

2. Set Text color to black;

6. Repeat step 5 for horizontal axis (if necessary).

7. Double-click on Baseline data series line on the graph (Figure 5);

1. In Series dropdown select Apply to all series;

1. Set Line thickness to 2;

2. Set Point size to $\mathbf{1 0 .}$

2. In Series dropdown select Baseline (or first series) (Fig. 6);

1. Set Color to Black;

3. Repeat previous step (7-2) for all other data series.

Horizontal (X) Axis Tick Mark Labels as Session Numbers (Choose One of the Following)

Every Session Tick Mark Appears and Has Labels ${ }^{4}$ (Fig. 7).

1. Double-click on horizontal (x) axis;

\footnotetext{
${ }^{1}$ You might need to select Use row 1 as headers and Use column $A$ as labels in Chart editor $>$ Setup.

${ }^{2}$ A legend might be required for multielement graphs. This is discussed later and this step can be skipped if you want to keep the legend.

${ }^{3}$ The Chart editor should appear whenever you double-click on parts of the graph and this is where the vast majority of formatting will occur.

${ }^{4}$ This allows the first session to be closer to the vertical (y) axis.
} 
Fig. 2 Insert a Line Chart from a Data Table. Note. This figure illustrates how to insert a new chart (i.e., line graph) in Sheets.

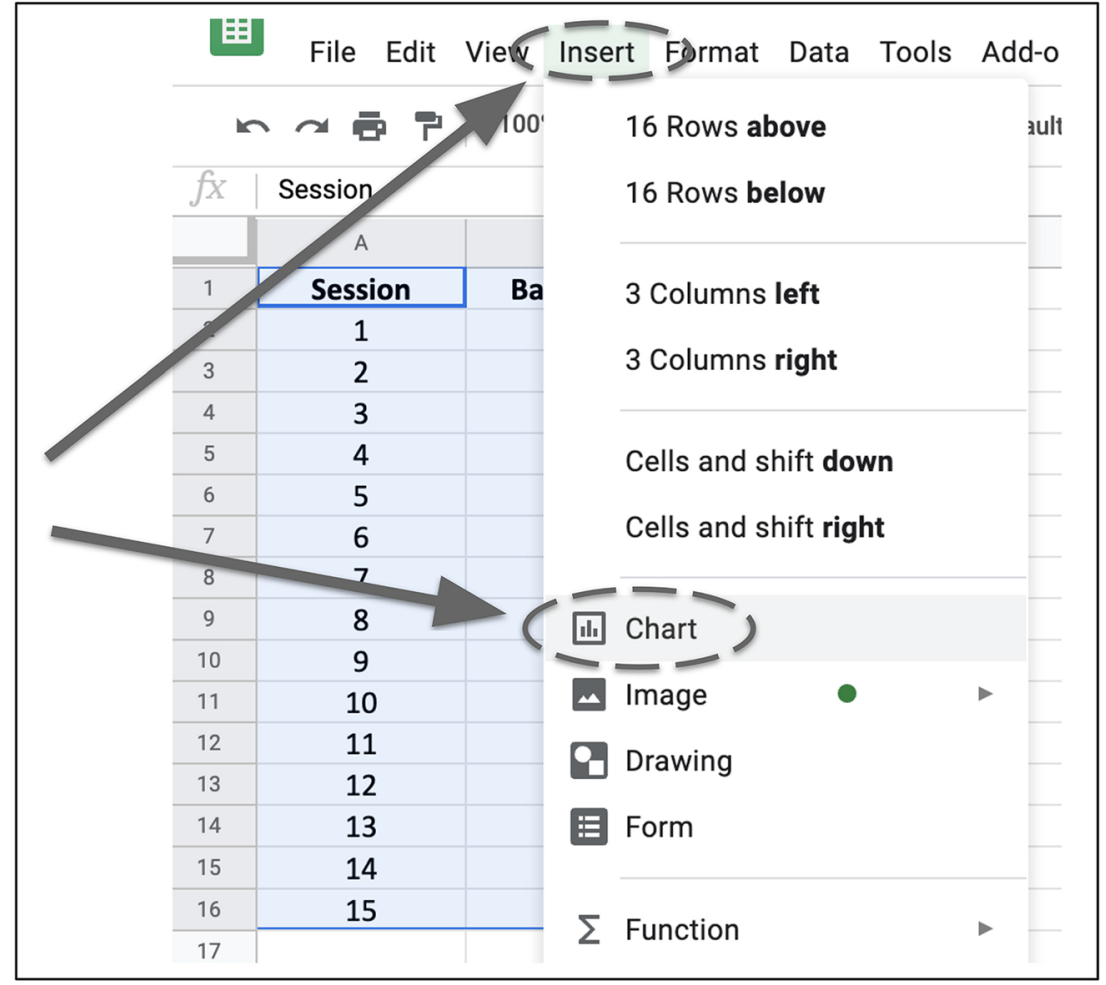

1. Select Treat labels as text;

Only Some Session Tick Marks Appear and Have
1. Double-click on horizontal (x) axis;

1. Deselect Treat labels as text;

2. Set Min. to 0;

Labels (Fig. 8).

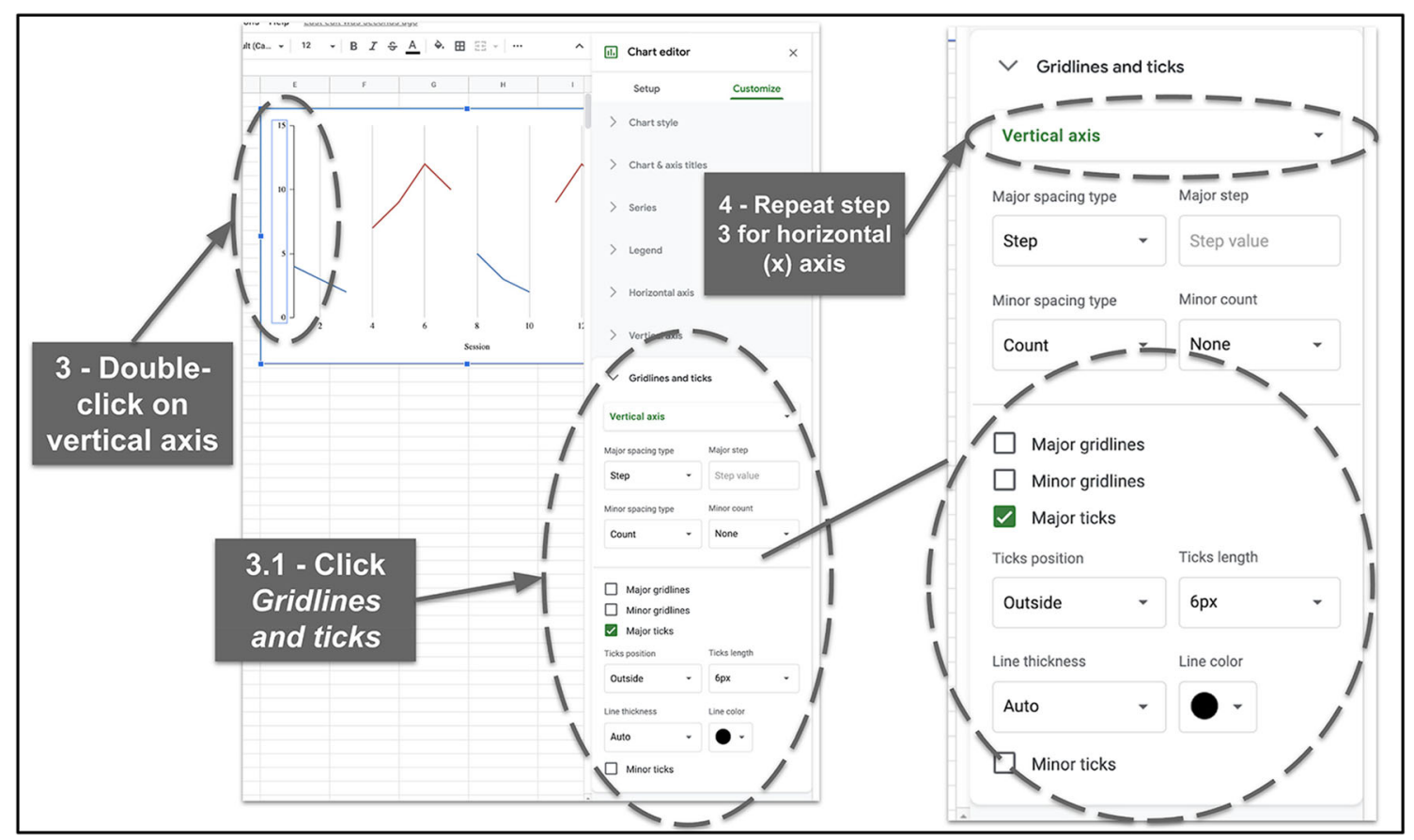

Fig. 3 Remove the Gridlines and Add Tick Marks. Note. This figure illustrates the steps for removing gridlines and adding axis tick marks in Sheets 


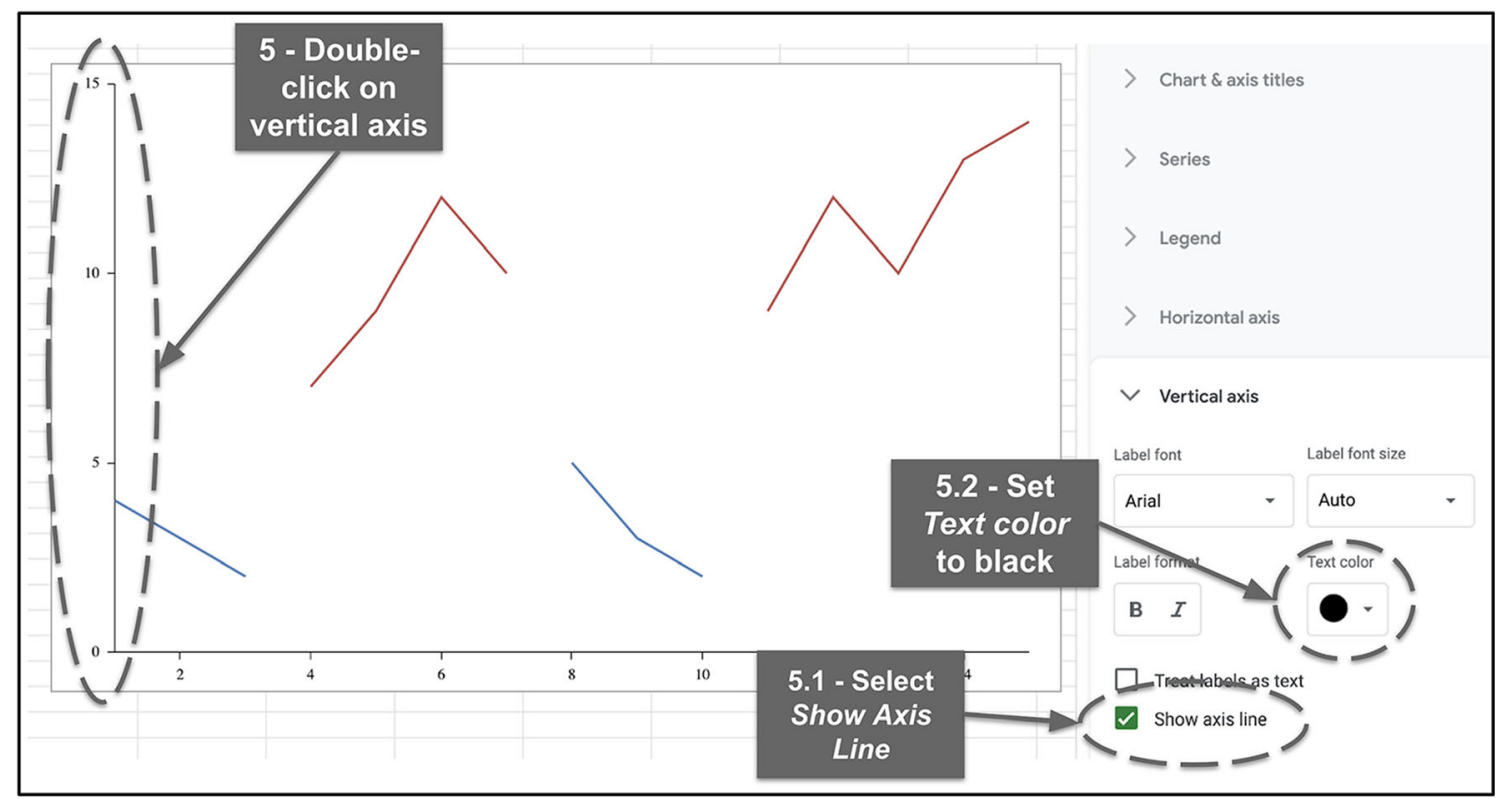

Fig. 4 Format the Axis Lines. Note. This figure illustrates the steps for formatting the vertical (y) and horizontal (x) axes

3. Deselect Allow bounds to hide data ${ }^{5}$

2. Click Gridlines and ticks;

1. Set Major spacing type to Step;

2. Set Major step to preferred tick mark label interval/period;

3. Cover up the $\mathbf{0}$ session number in Slides.

\section{Horizontal (X) Axis Tick Mark Labels as Dates (Choose One of the Following)}

Every Date Tick Mark Appears and Has Labels ${ }^{6}$ (Fig. 9).

1. Enter dates in Session column of data table (instead of session numbers);

2. Double-click on horizontal (x) axis;

1. Select Treat labels as text;

2. Set Slant labels to Auto. ${ }^{7}$

\footnotetext{
5 Directions for hiding the excess axis line and the zero session number are explained in a subsequent section.

${ }^{6}$ When creating a new date-based graph it is recommended to create a new graph from scratch to ensure that all formats and settings are set to default values before proceeding.

${ }^{7}$ Depending on the number of dates you may now need to enlarge the graph to ensure that all dates are displayed horizontally
}

Only Some Date Tick Marks Appear and Have Labels (Fig. 10).

1. Enter dates in Session column of data table (instead of session numbers);

2. Double-click on horizontal (x) axis;

1. Deselect Treat labels as text;

2. Set Slant labels to Auto;

3. Click Gridlines and ticks;

1. Set Major count to preferred value (adjust based on number of dates in data table).

\section{Choose One of the Following}

Every Vertical (Y) Axis Tick Mark Appears and Has a Label (In General, This Moves the Floating Zero Farther from the Horizontal (X) Axis) (Fig. 11)

1. Double-click on vertical (y) axis;

1. Select Treat labels as text;

2. Set Min to a negative number (will vary depending on range - adjust until "floating" zero appears on graph) and set Max to desired maximum value;

3. Deselect Allow bounds to hide data.

\section{Click Gridlines and ticks}

1. Set Major spacing type to Step;

2. Set Major step to $\mathbf{1}$ (might need to return to step 1.2 to set the Min value again for "floating" zero to appear);

3. Hide/cover up the negative $y$ value in Slides (described later). 


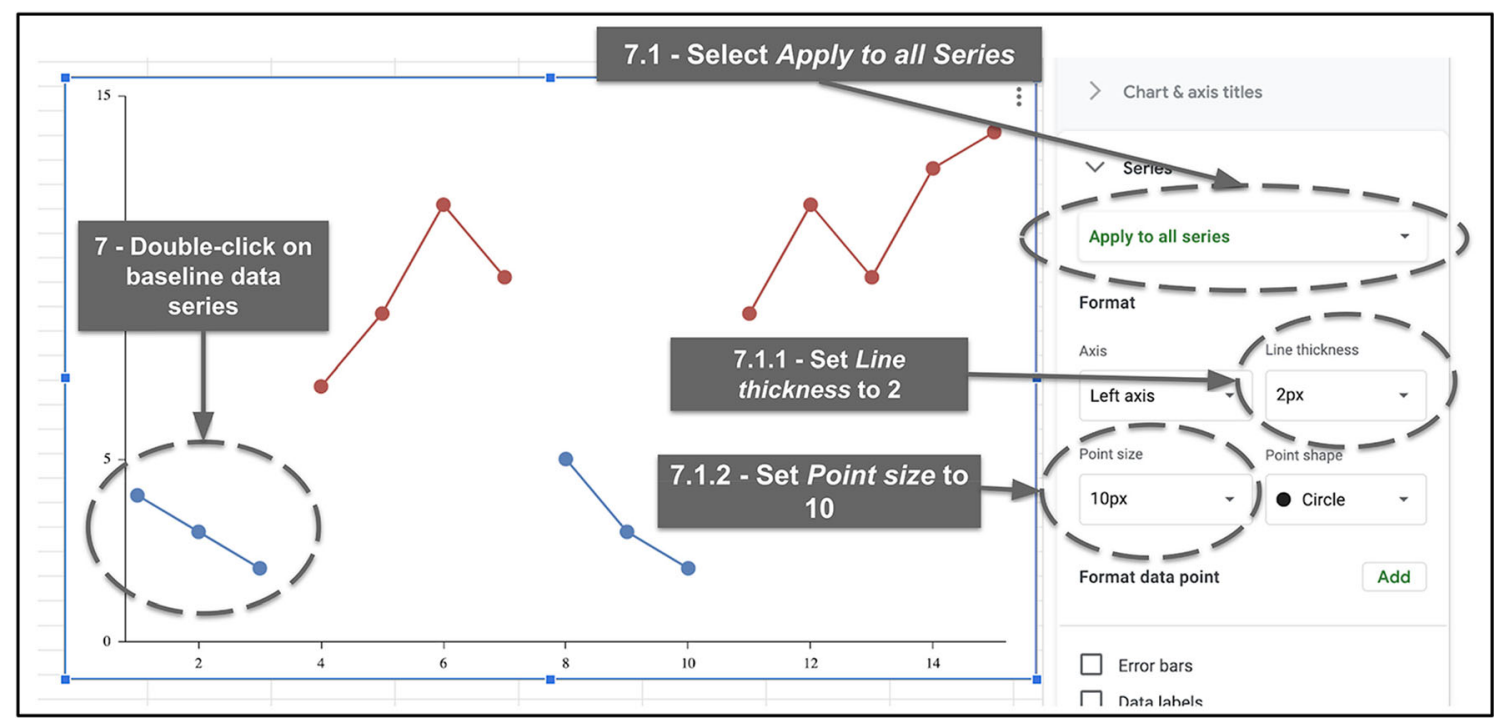

Fig. 5 Format the Data Series Lines and Data Points (Part 1 of 2). Note. This figure illustrates the steps for formatting data series lines and data points (Part 1 of 2) in Sheets

Only Some Vertical (Y) Axis Tick Marks Appear and Have Labels (In General, This Allows the Floating Zero to Be Closer to the Horizontal (X) Axis) (Fig. 12)

1. Double-click on vertical (y) axis;

1. Select Treat labels as text;

2. Set Min to a negative number (will vary depending on range - adjust until "floating" zero appears on graph $)^{8}$ and set Max to desired maximum value;

3. Deselect Allow bounds to hide data.

\section{Click Gridlines and ticks}

1. Set Major spacing type to Count;

2. Set Major count to Auto (might need to return to step 1.2 to set the Min value again for "floating" zero to appear).

\section{Adding More Data}

To add more data, add new rows to the bottom of the data table. In most cases, Sheets will not automatically add the data to the chart simply by adding a new row. To add the new data from the table to the graph:

1. Double-click on the graph then click on Setup in the Chart editor.

\footnotetext{
$\overline{8}$ Google Sheets appears to behave inconsistently here and you might need to readjust the Max value at this point-you might need to increase the maximum value then revert back to original preferred max for the floating zero to "look good."
}

1. Change the Data range to include the newly added rows.

\section{Adding a New Data Series Column (e.g., condition/phase) to Preexisting Graph}

To add a new condition/phase (i.e., data series) to a preexisting graph, add the new columns of data, with column headers, to the preexisting data table. Adding a new column of data will most likely not automatically add the data to the graph and you will need to add the new data series manually. To add the new data from the table to the graph:

1. Double-click on the graph then click on Setup in the Chart editor.

1. Change the Data range to include the newly added columns;

2. Click on Add series and select the new series to be added ${ }^{9}$

3. Format the line and data points for the newly added data series.

\section{Specific Formatting for Multiple Baseline Design Graphs (Fig. 13) ${ }^{10}$}

\section{Duplicate Graphs}

To maintain formatting across all tiers in a multiple baseline research design graph, consider creating the

\footnotetext{
9 Make sure you added a column header prior to adding the data to the graph.

${ }^{10}$ See Carr (2005) for further design considerations when creating multiple baseline design graphs.
} 


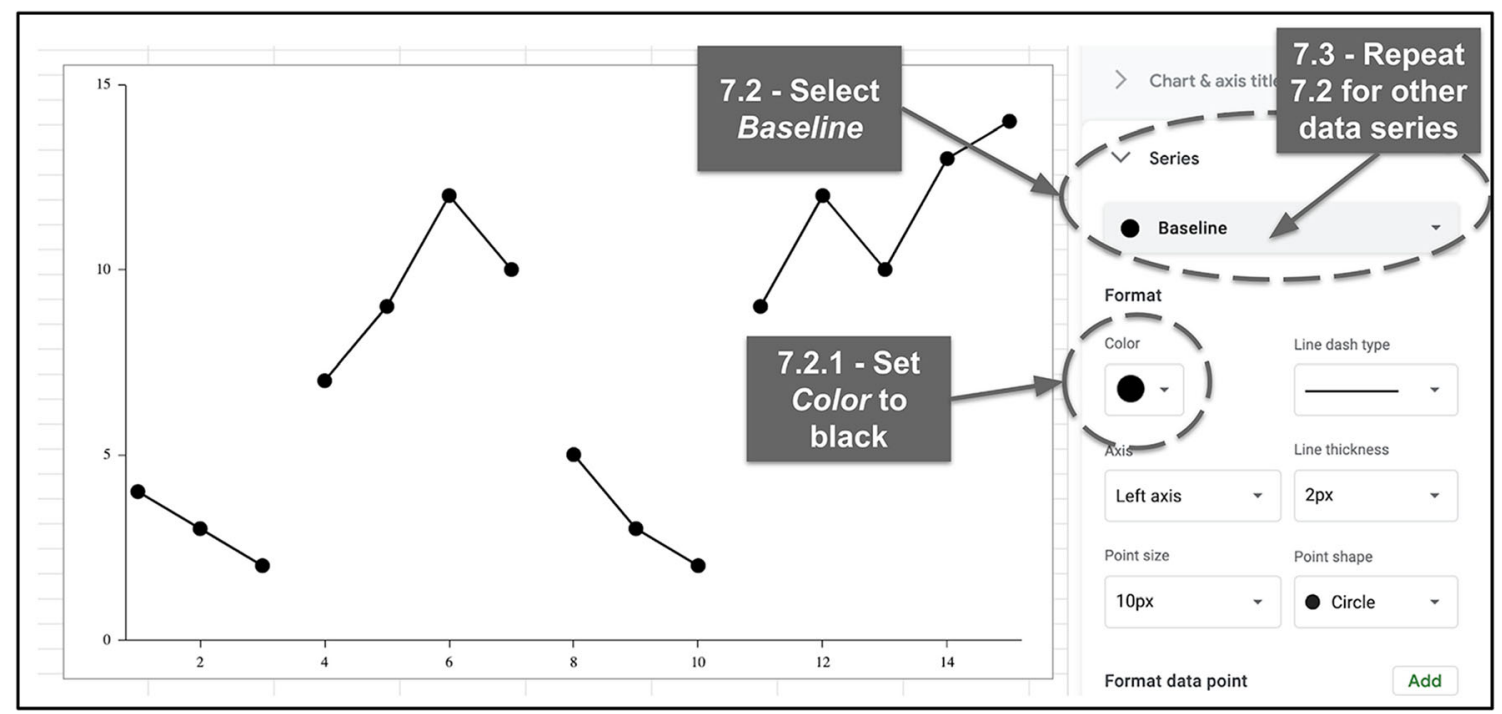

Fig. 6 Format the Data Series Lines and Data Points (Part 2 of 2). Note. This figure illustrates the steps for formatting data series lines and data points (Part 2 of 2) in Sheets

bottom/last tier first then duplicate that completed graph (with all desired formatting, sizing, etc.) for subsequent upper tiers.

\section{Font Sizes in Sheets}

Given that two or more graphs will be stacked on a single portrait-oriented page in Slides, and the graphs themselves will likely appear smaller than other graphs, consider increasing the size of the font for the vertical (y) and horizontal (x) axes tick mark labels at this point.

\section{Same Number of Sessions/Dates and Vertical (Y) Axis Range}

Ensure that the horizontal (x) axes for each tier in a connected multiple baseline design graph have the same number of sessions or the same dates and ensure that the vertical (y) axis has the same range, step count, etc. (if necessary).

\section{No Horizontal (X) Axis Tick Mark Labels for Top Tier(s) of Multiple Baseline Design Graphs (Fig. 14)}

1. Double-click on horizontal axis;

2. Change Text color to White.

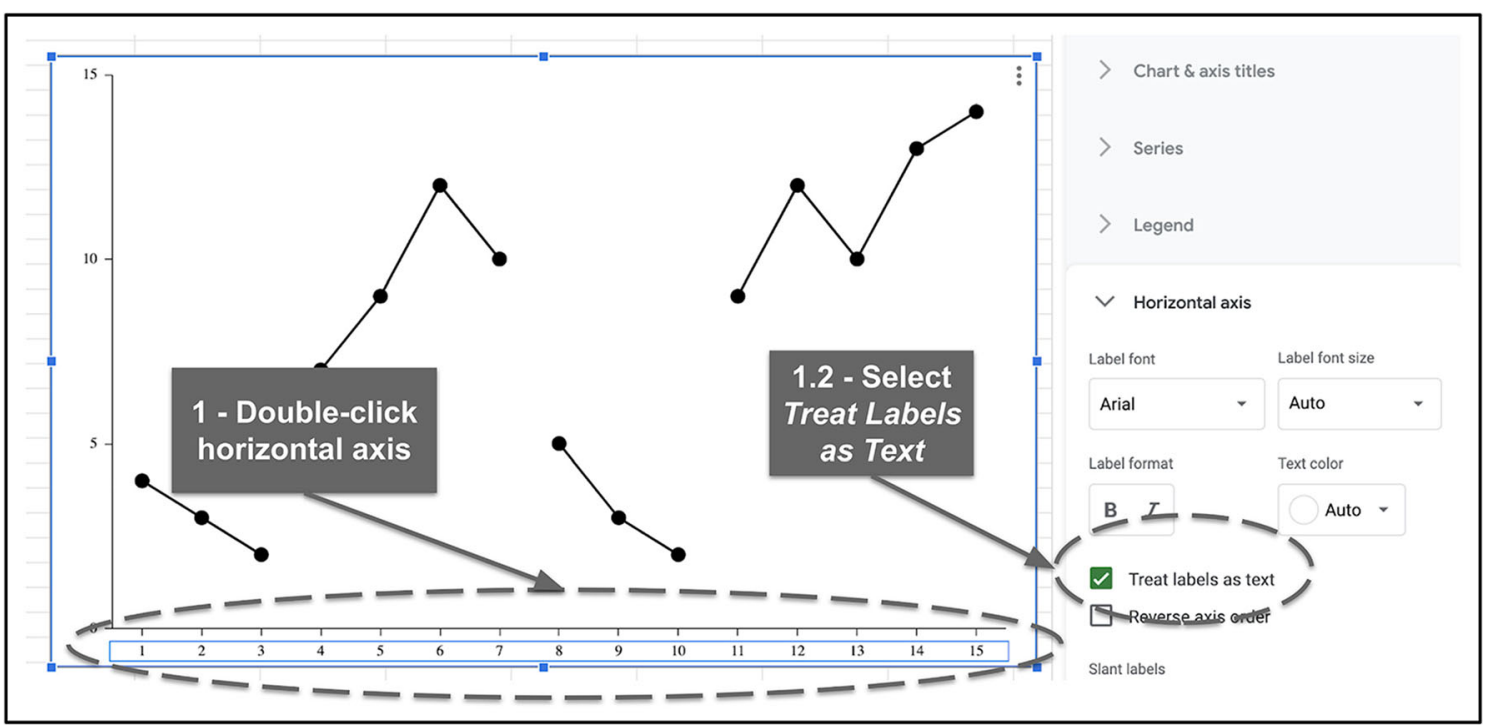

Fig. 7 Make Tick Marks and Labels Appear for Every Session. Note. This figure illustrates the steps to ensure that horizontal (x) axis tick marks and labels appear for every session in Sheets 


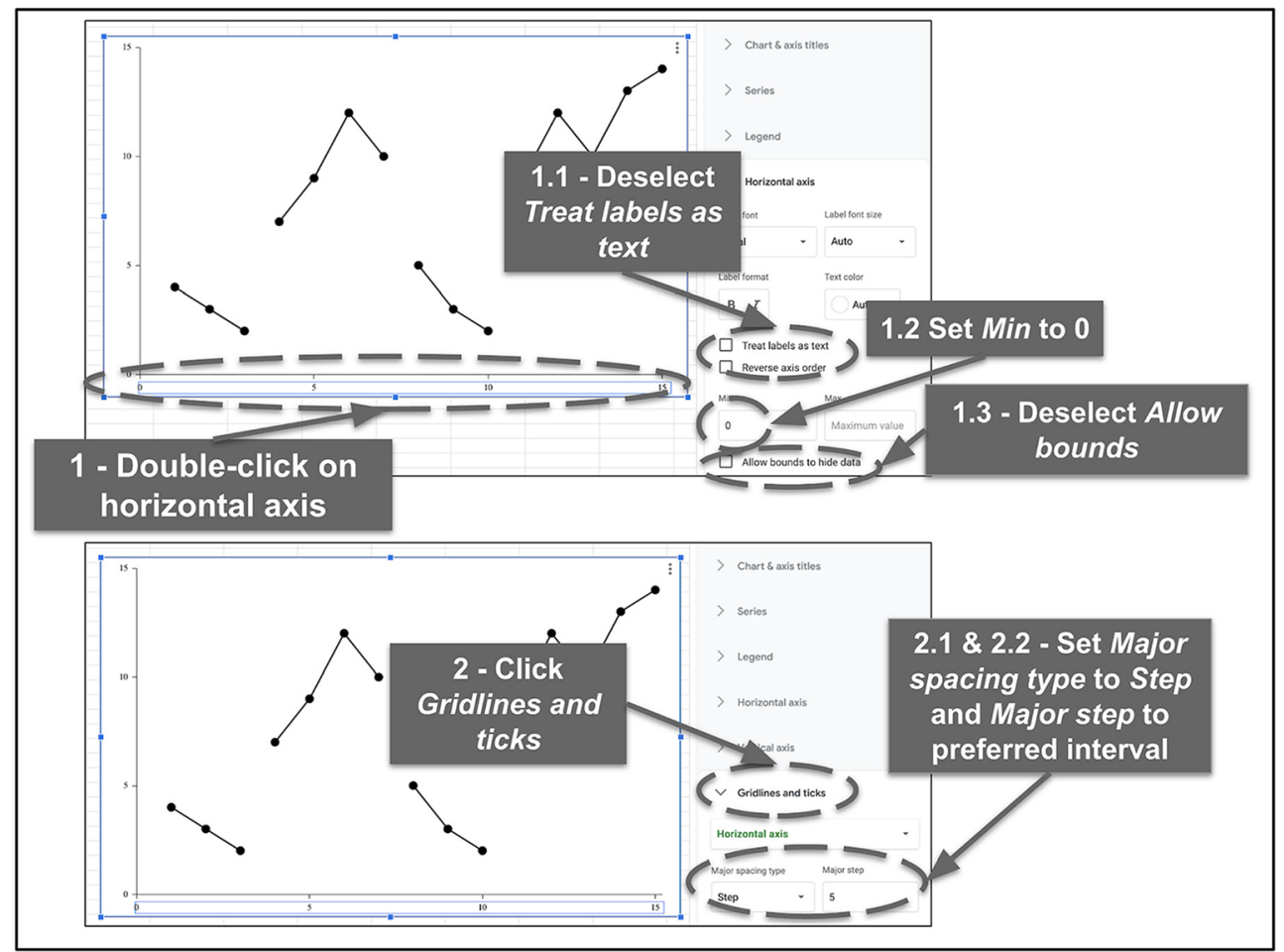

Fig. 8 Make Tick Marks and Labels Appear for Only Some Sessions. Note. This figure illustrates the steps to ensure that horizontal (x) axis tick marks and labels only appear for some sessions in Sheets.

\section{Specific Formatting for Multielement (Alternating Treatments) Design Designs}

To create a multielement design graph:
1. If the data series markers are not different shapes, change them by double-clicking on each data series line and selecting different point shapes in the Chart editor).

2. Click Customize in the Chart editor and then click Legend and insert the legend where desired.

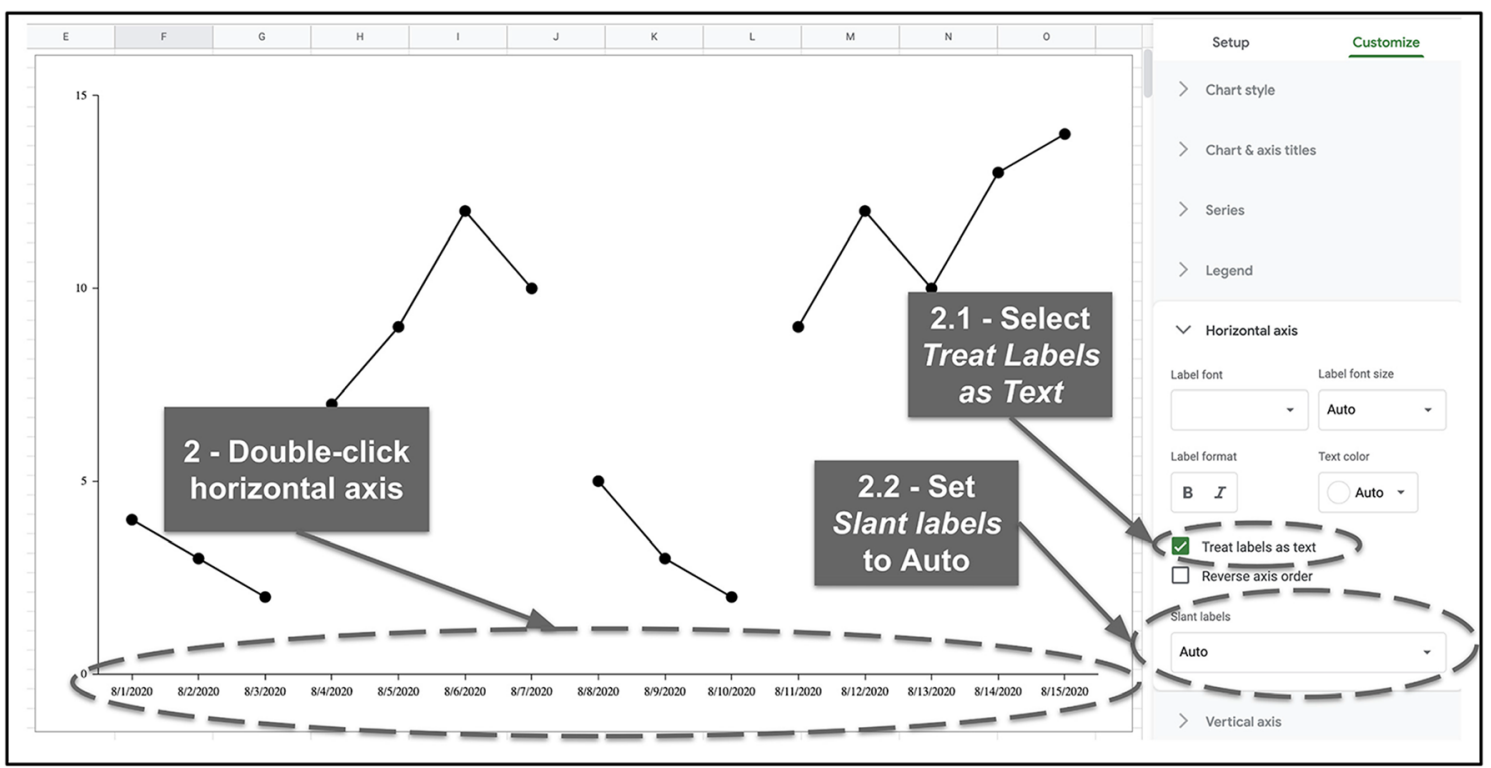

Fig. 9 Make Tick Marks and Labels Appear for Every Date. Note. This figure illustrates the steps to ensure that horizontal (x) axis tick marks and labels appear for every date in Sheets 
Fig. 10 Make Tick Marks and Labels Appear for Only Some the steps to ensure that horizontal (x) axis tick marks and labels appear for only for some dates in Sheets Dates. Note. This figure illustrates

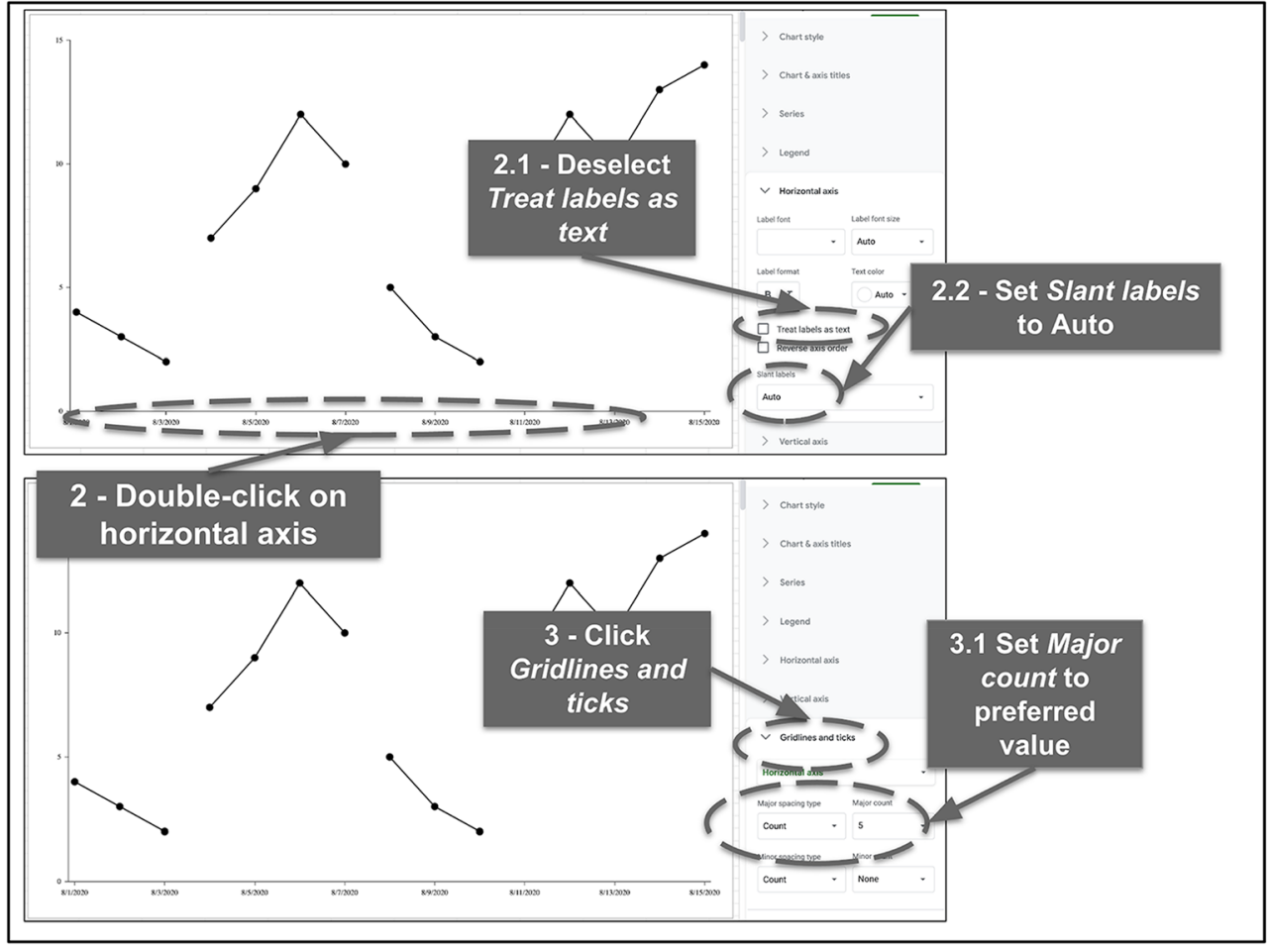

\section{Final Formatting Before Making Copies of Graphs and/or Linking Graphs to Slides}

As a reminder, it is advised to use the completed graph as a template for subsequent graphs in Sheets; therefore, ensure that all formatting has been finalized. In addition, given that a dynamic image of the graph will be linked to Slides, whereas the data in the graph will change, the formatting will no longer be editable within Slides itself and thus must be conducted now. At this

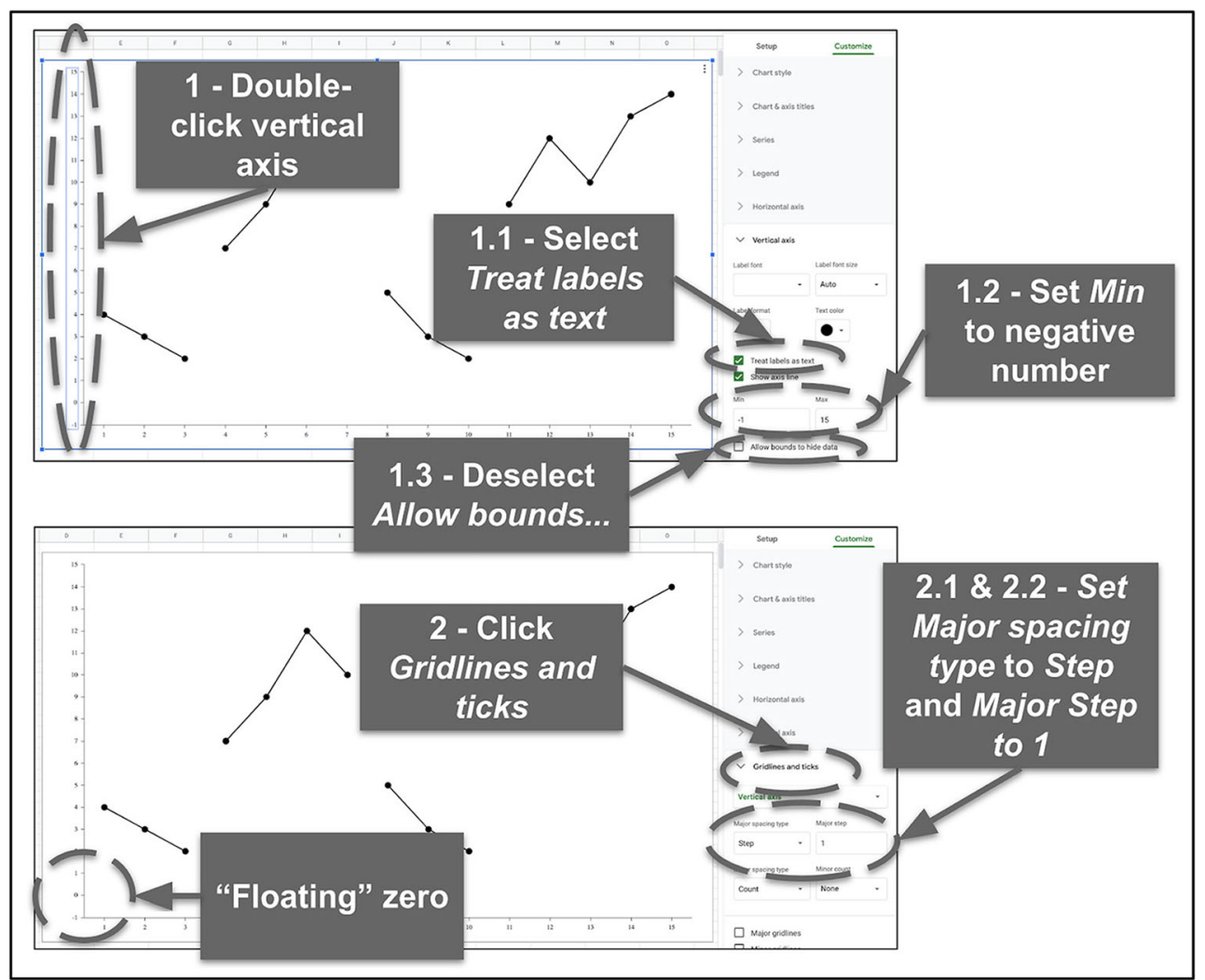

Fig. 11 Make Tick Marks and Labels Appear for Every Vertical (Y) Axis Increment. Note: This figure illustrates the steps to ensure that vertical (y) axis tick marks and labels appear for every increment in Sheets 
point, consider changes to font styles and sizes as well as the following:

1. If the maximum vertical (y) axis or horizontal (x) axis data point isn't showing or is partially hidden:

1. Set the max for that axis to greater than the desired maximum value and then hide the excess axis line in Slides (discussed later).

2. If either axis line is now too long, reduce maximum value in the range (if possible without hiding maximum data point [s]).

3. Decide whether the axis titles will be included or removed (you can add these titles later in Slides or delete the titles to use the individual graphs in multiple baseline design graphs). To remove the axis titles, click on the title and press delete.

4. Ensure that the aspect ratio of the graph is suitable for copying, downloading, presenting, adding to submitted manuscript, printing, and/or resizing in other applications. Ensure same ratio/size for each tier in linked multiple baseline graphs.

\section{Making a Copy of a Graph for Reuse within Sheets}

To preserve the formatting, size, and aspect ratio of the first graph across multiple similar graphs, duplicate the current tab in sheets (right-click on current tab and select Duplicate) and then edit the data table in the new sheet. This method can be used for creating a multiple baseline design graph or for creating several withdrawal or multielement graphs for the same research project or manuscript. As previously noted, use caution when copying or duplicating graphs within sheets and if desired formatting cannot be obtained, we suggest creating a new tab/file and starting from scratch for new graphs.

\section{Using Slides for Final Formatting and Presenting}

Several of the following formatting elements can be accomplished in Sheets, however, doing so is more challenging because Sheets has limited graphical editing and formatting capabilities. For example, creating, inserting, and moving objects like text boxes and phase/condition lines in Sheets is much more cumbersome and less functional than performing the same task in Slides. Another example where formatting is far easier in Slides is when creating multiple baseline design graphs where precise formatting and visual alignment are required. In addition, Slides is a visual presentation application with a primary function of displaying images in a visually appealing way, whereas this function is secondary in Sheets. The additional step of copying, pasting, and linking a graph from Sheets to Slides allows for a much more usable and professional final product in Slides. Similar steps can be conducted in Google Docs, however, like Sheets, Docs is not primarily a graphical editing or presentation application, and thus the final product will not be as visually appealing as the product created in Slides.

Copy and Link Chart ${ }^{11}$ from Sheets to Slides (Fig. 15)

1. In Sheets, click the three-dot menu in upper right of chart and select Copy chart;

2. Open new Slides file;

3. In Slides, on an empty slide, right-click and click Paste. Select Link to spreadsheet then click Paste.

To have multiple graphs with the same dimensions and aspect ratios, after making final edits to the graph in Sheets, do NOT change the chart size in Sheets before copying to Slides. Set the size once in Sheets, then perform all final resizing in Slides, treating the linked graph as a single image in Slides without changing the aspect ratio in Slides (i.e., resize the graph in Slides by dragging the corner of the image or with Format options).

\section{General Formatting of Linked Chart in Slides $^{12}$}

\section{Phase/Condition, Axes, and Data Series Labels (Figure 16)}

Insert condition, axes, or data series labels by clicking the insert text box tool and then type the name of the label. Center the condition label above the condition on the graph. Use the automated red/blue alignment lines to align the text boxes for different conditions with each other. Place the data series label text box near a data point and insert an arrow pointing from the text box to the data point (if applicable).

\section{Phase/Condition Lines (Fig. 17)}

Insert phase/condition lines using the insert line tool and place the line on the graph in the appropriate location.

\footnotetext{
${ }^{11}$ Linking charts from Sheets to Slides ensures that the chart in Slides is automatically updated based on changes made in Sheets.

${ }^{12}$ Pro tip: Use formatting guides in Slides to help align objects. Click View, hover on Guides, click Show Guides.
} 


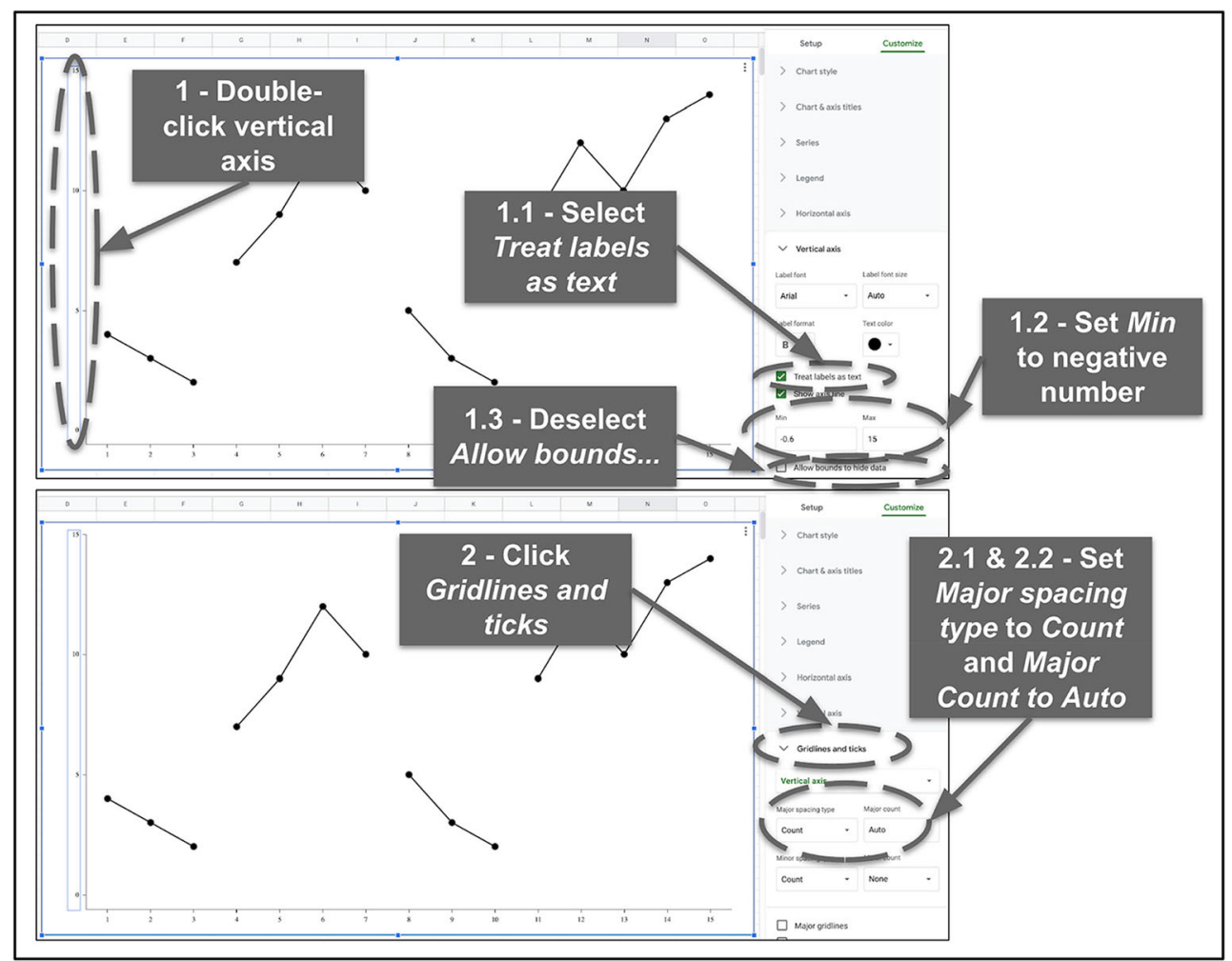

Fig. 12 Make Tick Marks and Labels Appear for Only Some Vertical (Y) Axis Increments. Note. This figure illustrates the steps to ensure that vertical (y) axis tick marks and labels only appear for some increments in Sheets

\section{Hiding (Covering) Extraneous Parts of a Graph (e.g., Negative Axis Values, Extra Axis Line Length) (Figure 18)}

Hide/cover-up negative axis values or extraneous axis line lengths by creating a white box with no borders and place the white box where needed on the graph. Click the insert shapes tool and place the box over the area of the graph that needs to be hidden. Edit the box to change the color of the box to white and change the color of the border to transparent/ white.

\section{Specific Formatting for Multiple Baseline Design Graphs}

\section{Change Slide Page Orientation to Portrait}

To create a vertically stacked composite multiple baseline design graph it is advised to change the orientation of the slide to Portrait (as opposed to the default landscape):

1. Click File and select Page setup;

2. Click Custom;

3. Set dimensions to 8.5 in by 11 in.

\section{Distribute Graphs Vertically ${ }^{13}$}

To ensure that each tier is spaced the same distance from each other vertically, highlight all three graphs, right-click and click Distribute vertically.

\section{Tier Labels}

Create tier labels as you did for other labels by creating and placing text boxes. However, for tier labels, set the border color to black. Place the tier label in the desired location on one graph and place the other tier labels in the same place on their respective graphs and use the alignment guides in Slides to align the text boxes across graphs.

\section{Centered Vertical (Y) Axis Label}

Create a single vertical (y) axis label as you have done before by creating a borderless text box. Rotate the text box so that it is vertical and place the text box centered on the composite multiple baseline design graph.

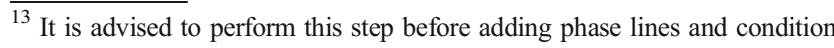
labels.
} 


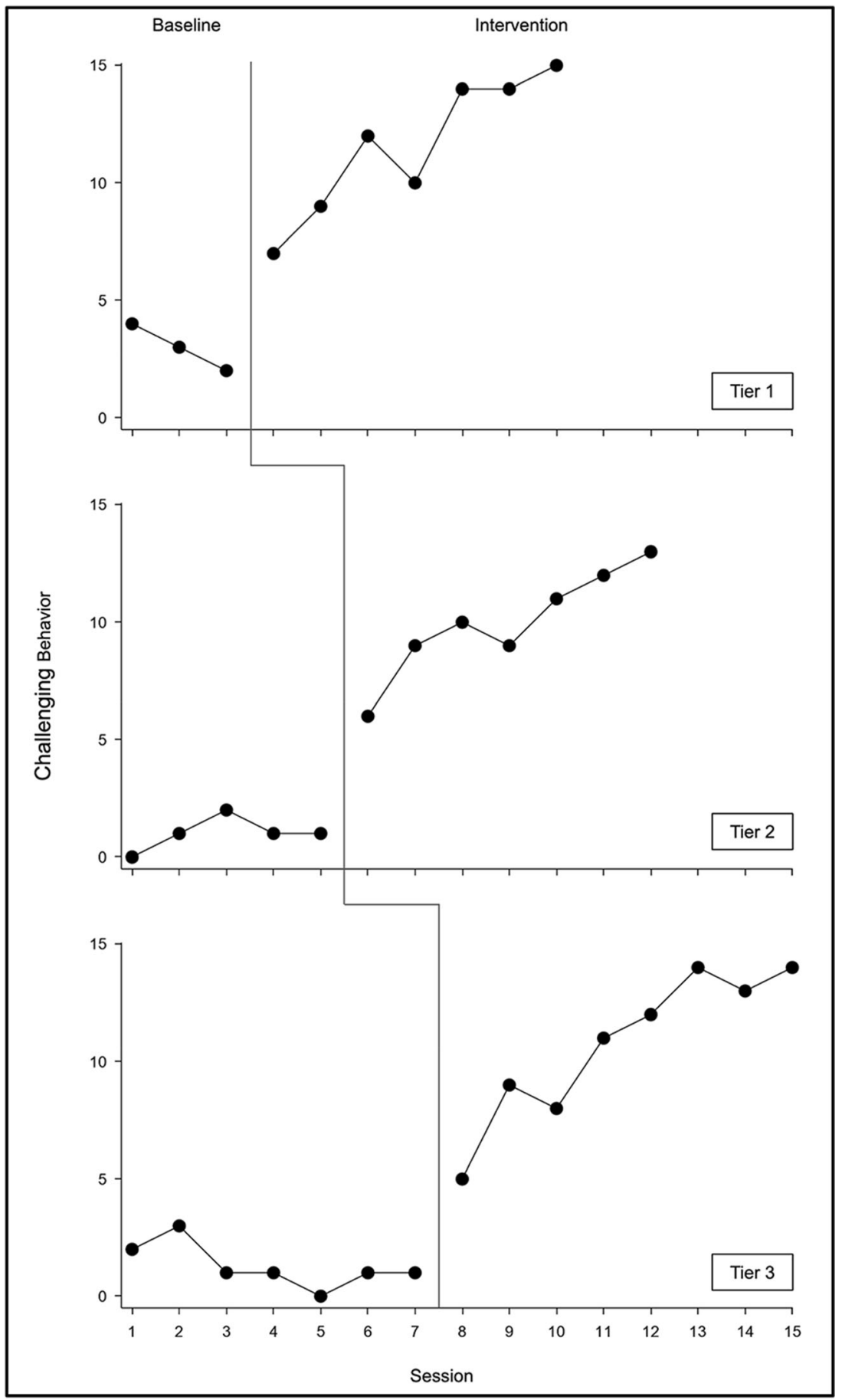

Fig. 13 An Example of a Multiple Baseline Research Design Graph Created in Sheets and Slides. Note. This figure presents a multiple baseline design graph with preferred characteristics and formatting created in Sheets and Slides

\section{Phase/Condition Change Lines}

Create phase/condition change lines as you have done before. However, for multiple baseline design graphs, the phase change lines must be connected across tiers. Inserting and aligning phase change and connecting lines might be the most challenging step in the process of creating multiple baseline design graphs and practice, a lot of trial and error, and plenty of patience will most likely be necessary to align the lines as desired. To assist with line sizing and alignment, zoom in on sections of the graph: click View then Zoom then 200\%. In addition, to keep lines perfectly horizontal or vertical when resizing or rotating, hold down Shift when using the mouse to rotate or resize lines. Also, holding down Shift while moving the lines (or any other object) with the keyboard arrows will allow for a possibly more precise placement of the lines. 


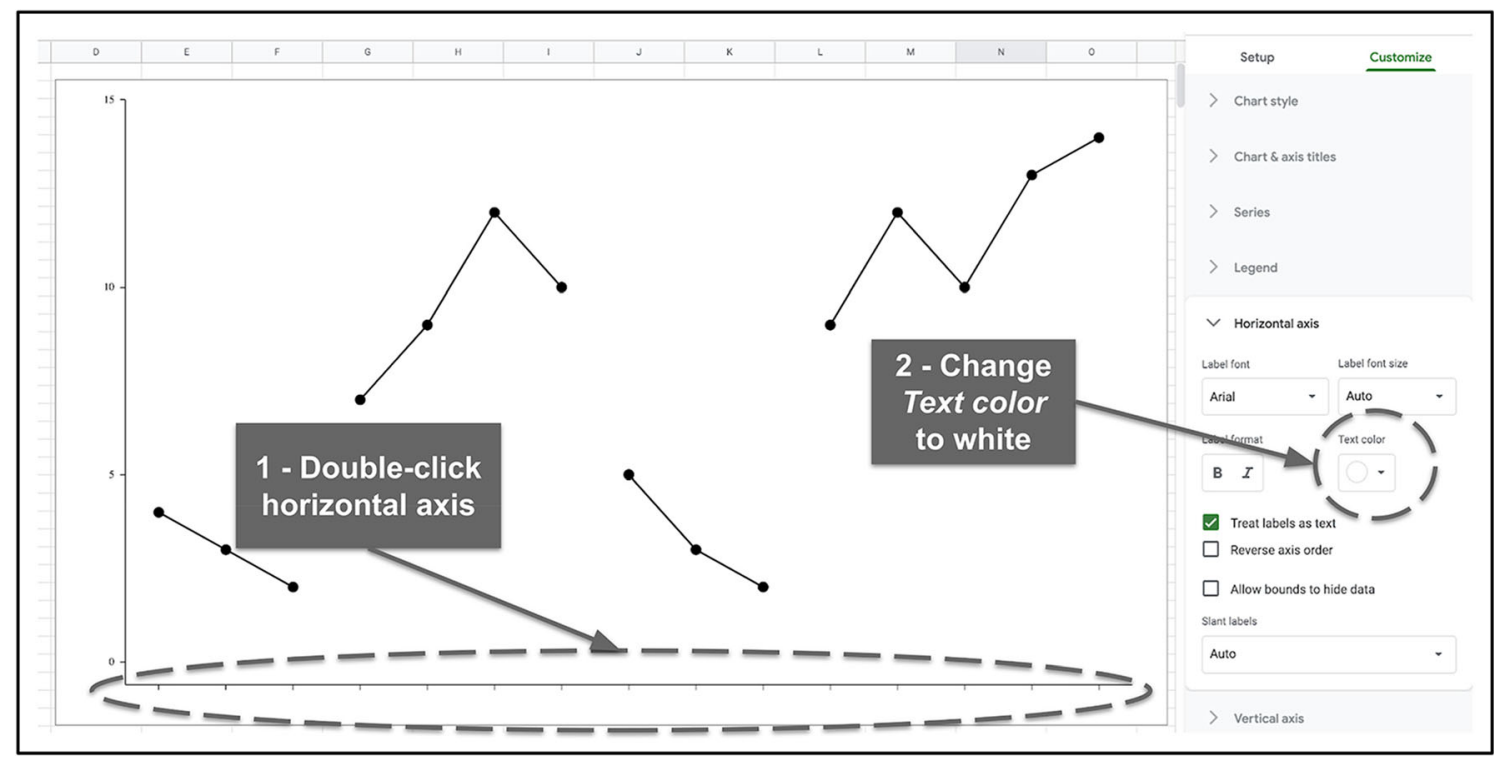

Fig. 14 Remove the Horizontal (X) Axis Tick Mark Labels. Note. This figure illustrates the steps to remove the horizontal (x) axis labels in Sheets for a multiple baseline design graph

\section{Discussion}

We hope that the preceding task analysis for creating a variety of single-subject research design graphs in Sheets and Slides will prove to be helpful to clinicians and researchers who are looking for a simple, minimalistic, free, and easy-to-use graph production system. Although the system described here meets many of the needs of ABA practitioners, there are several limitations to note.
One limitation that might be frustrating for some behavior analysts is the inability to create unfilled data markers (e.g., open circles, open squares) in Sheets. We agree that this is a substantial limitation when compared to other graph creation applications. A limited workaround would be to change the color of every individual data point to white (the connecting lines will still appear) in the graph Chart editor in Sheets, link the graph to Slides, then create unfilled shapes in Slides and place those shapes on top of the linked graph where the data markers would

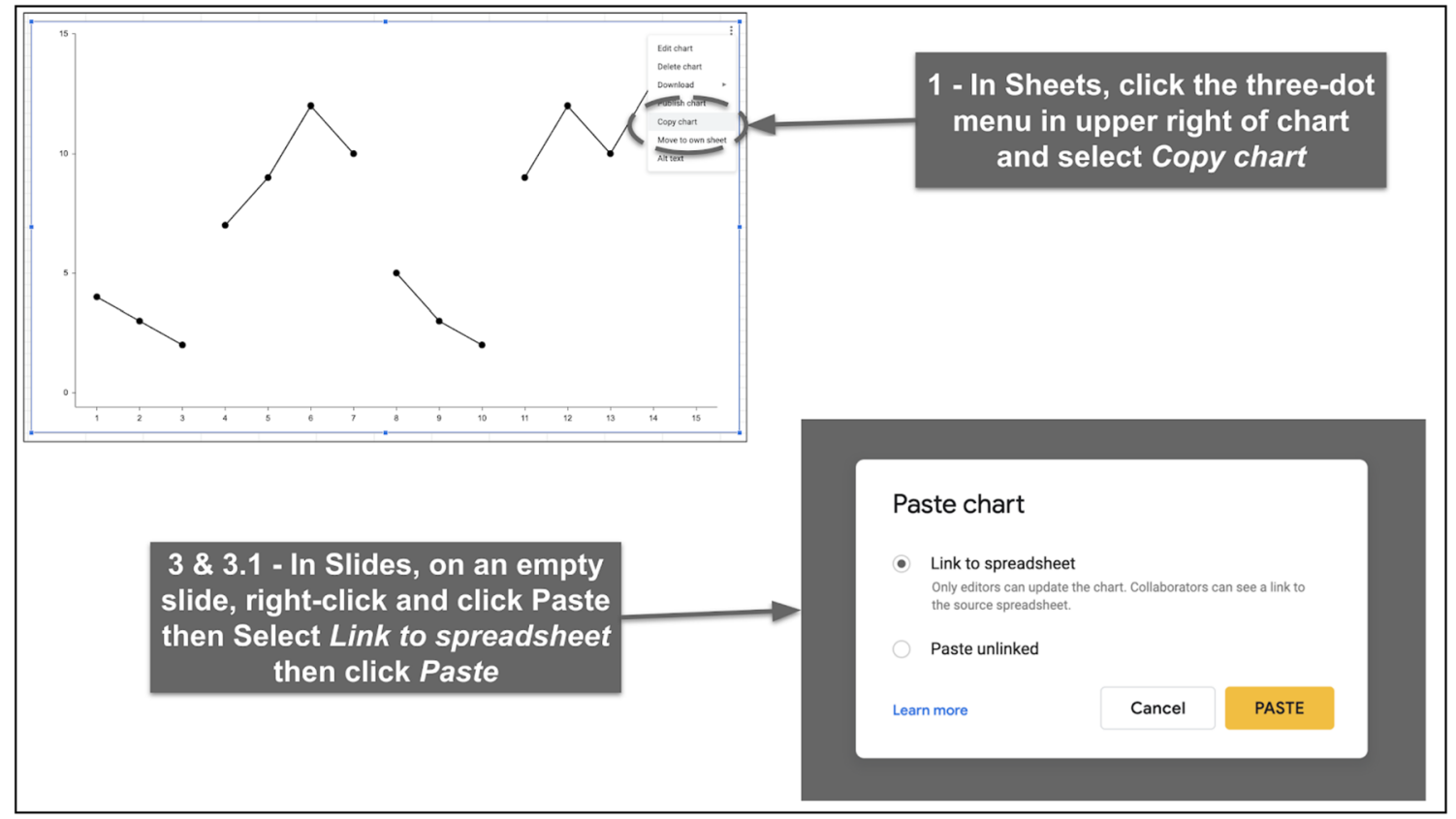

Fig. 15 Copy and Link Graph from Sheets to Slides. Note. This figure illustrates the steps to copy and paste a graph from Sheets to Slides, and how to link the graph to Slides from Sheets 


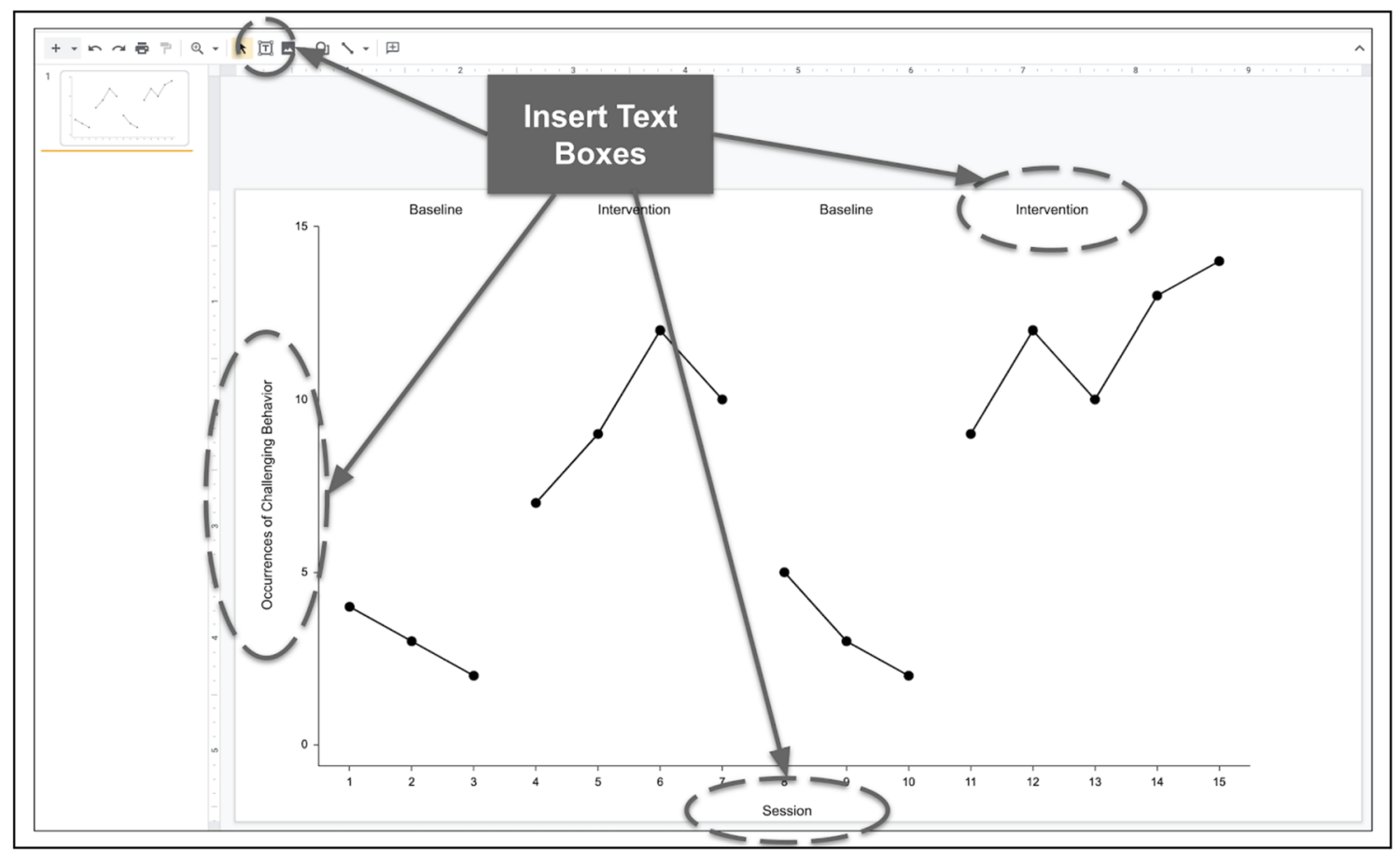

Fig. 16 Add Labels to the Linked Graph in Slides. Note. This figure illustrates the steps for inserting phase/condition and axis labels to a graph in Slides

normally have appeared. This is not an ideal solution, but currently unfilled data markers are not an option in Sheets.

Unlike other graphing tutorials (Dubuque, 2015; Fuller \& Dubuque, 2018), our tutorial does not provide instructions for incorporating automatically generated and updated phase change lines in Sheets. Given the intended audience of this introductory tutorial, we elected to not include this option; however, manually inserting and updating phase change lines in Slides is fairly simple and not prohibitively time-consuming.

A series of related limitations to the system that we described include the fact that it requires the use of two applications, requires the user to be somewhat fluent with

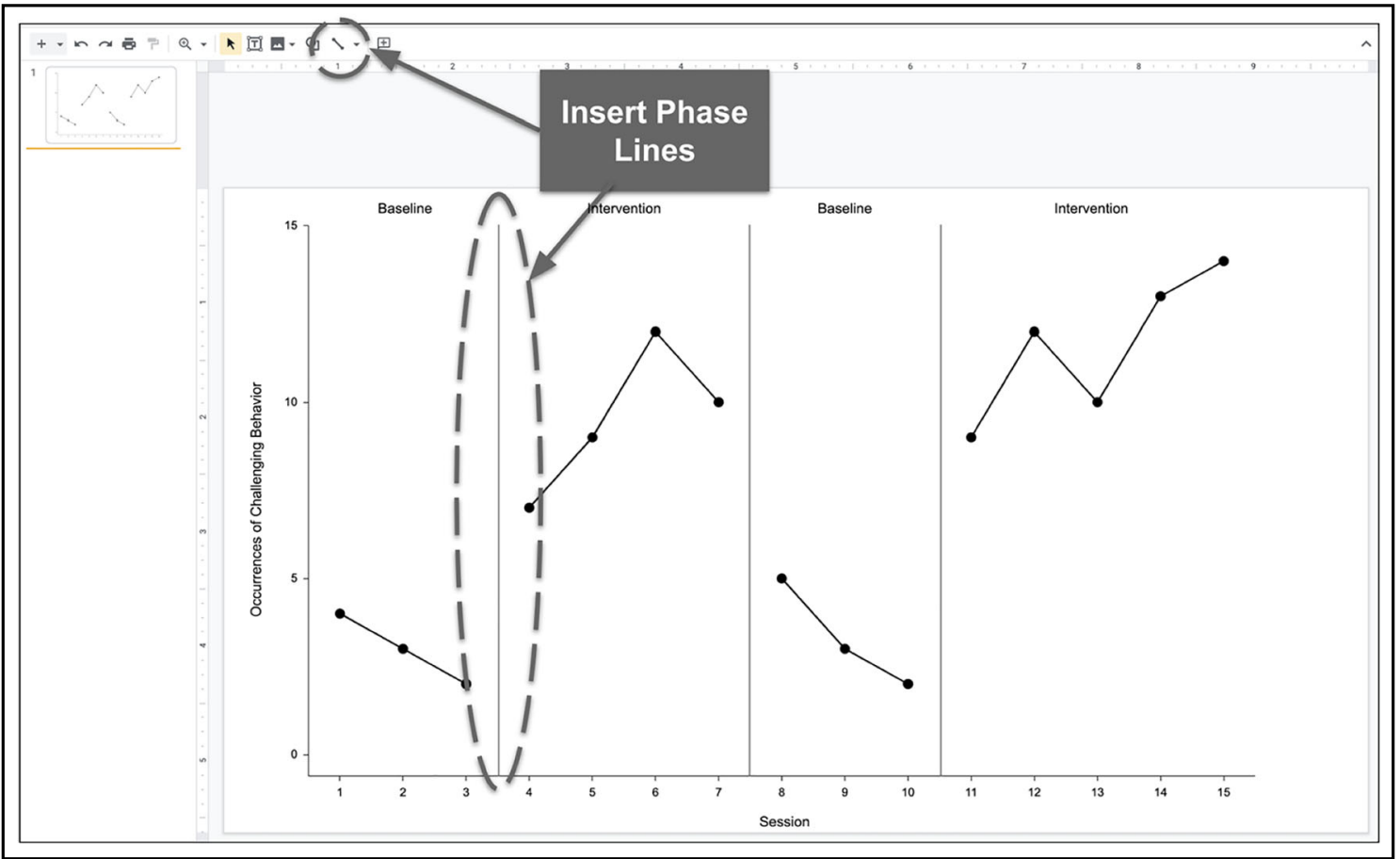

Fig. 17 Insert Phase/Condition Lines. Note. This figure illustrates the steps for adding phase/condition change lines to a graph in Slides 


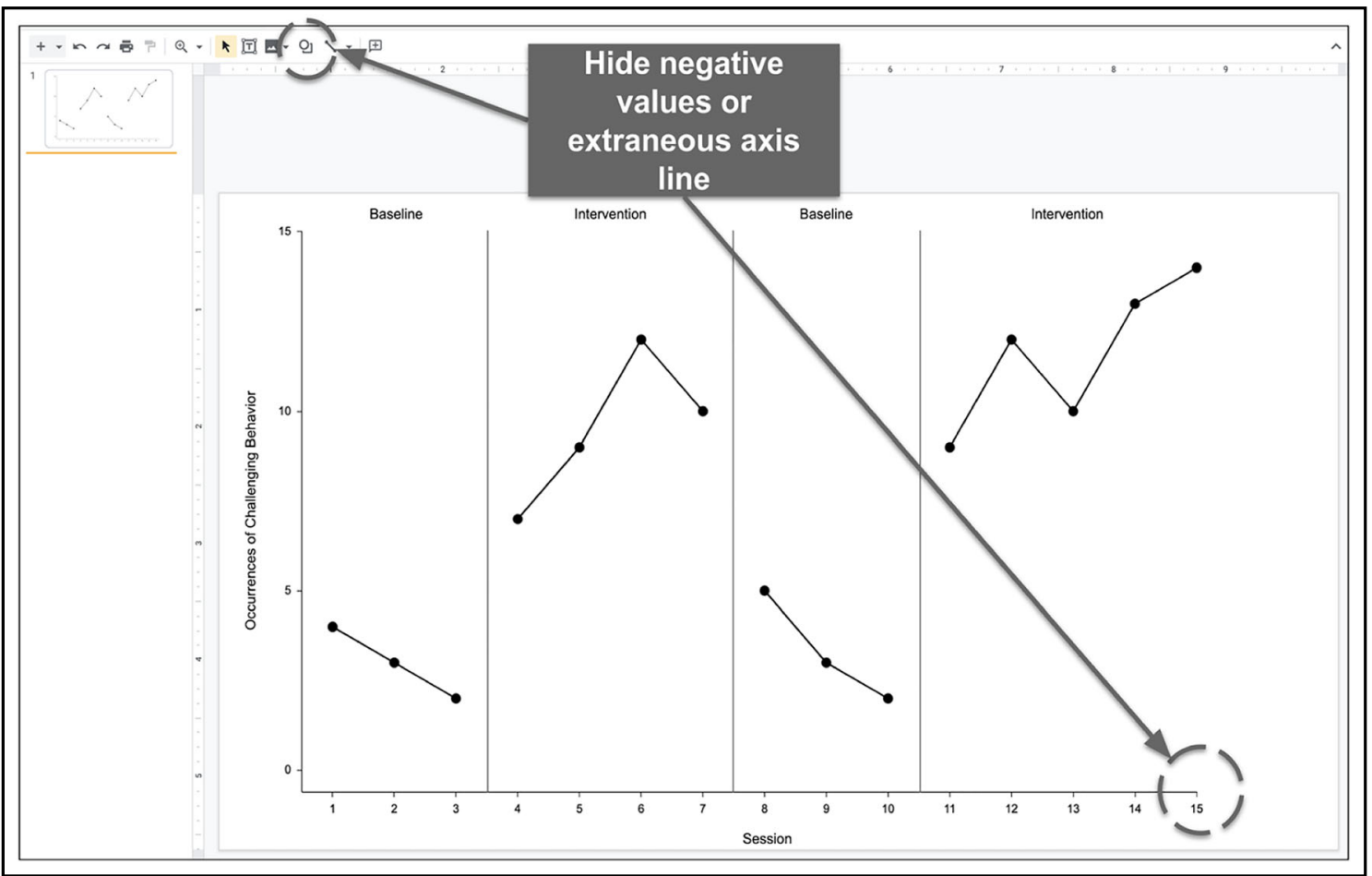

Fig. 18 Hide Parts of Graphs in Slides. Note. This figure illustrates how to hide unnecessary parts of a graph in Slides>

both Sheets and Slides, and requires the user to be able to interact with both in order to create simple graphs. Although publication-quality graphs can be created with other single-graphing applications (e.g., Excel, Prism, Numbers, SigmaPlot), those more advanced and robust applications are not free or are only available for use with specific operating systems, whereas Sheets and Slides are both free, are web-based, and graphs can be created, edited, and viewed in Sheets on macOS, Windows OS, and Chrome OS. In addition, the use of the linked system allows clinicians and researchers to use Slides in order to present a constantly updated graph as a properly formatted image across a variety of screens and media.

A limitation to any cloud-based storage and document collaboration system is how privacy is managed and protectedregulations dictated by FERPA and HIPAA laws, in particular. This concern is not specific to the graph creation and presentation system that we have described; however, a note of caution is warranted to ensure that all users are aware that there are inherent data privacy and security risks. Similar risks exist whenever one sends an email attachment of an Excel graph with FERPA/HIPAA protected data. Although users can access Sheets and Slides for no cost, it is important to note that the no-cost access to these applications does not allow for any type of additional HIPAA protections. If a user wishes to use Google applications with HIPAA protections, additional costs and contractual agreements with Google are likely necessary, depending on individual circumstances. Regardless of the software application that a person uses, it is the user's responsibility to be aware of potential security risks and to comply with all regulations.

The preceding tutorial provided easy-to-follow steps to generate graphs for use in ABA research and practice. It is important to note that, over time, it is likely that Google may make adjustments to the Sheets and Slides applications and corresponding features and options; however, we hope that the instructions herein will provide the reader with sufficient skills to not only generate high-quality graphs but also, with providing familiarity with the many features included in the applications, generalize those skills to future versions of Sheets and Slides, and prepare them for any updates to come.

\section{Declarations}

Ethical approval This article does not contain any studies or experiments with human participants or animals performed by any of the authors.

\section{References}

Berkman, S. J., Roscoe, E. M., \& Bourret, J. C. (2018). Comparing selfdirected methods for training staff to create graphs using Graphpad Prism. Journal of Applied Behavior Analysis, 52(1), 188-204. https://doi.org/10.1002/jaba.522.

Blair, B. J., \& Shawler, L. A. (2019). Developing and implementing emergent responding training systems with available and low-cost computer-based learning tools: Some best practices and a tutorial. 
Behavior Analysis in Practice, 13(2), 509-520. https://oi.org/10. 1007/s40617-019-00405-x.

Carr, J. E. (2005). Recommendations for reporting multiple-baseline designs across participants. Behavioral Interventions, 20(3), 219-224. https://doi.org/10.1002/bin.191.

Carr, J. E., \& Burkholder, E. O. (1998). Creating single-subject design graphs with Microsoft Excel ${ }^{\mathrm{TM}}$. Journal of Applied Behavior Analysis, 31(2), 245-251. https://doi.org/10.1901/jaba.1998.31-245.

Cooper, J. O., Heron, T. E., \& Heward, W. L. (2020). Applied behavior analysis. Pearson Education.

Dixon, M. R., Jackson, J. W., Small, S. L., Horner-King, M. J., Lik, N. M. K., Garcia, Y., \& Rosales, R. (2009). Creating single-subject design graphs in Microsoft Excel ${ }^{\mathrm{TM}}$ 2007. Journal of Applied Behavior Analysis, 42(2), 277-293. https://doi.org/10.1901/jaba.2009.42-277.

Dubuque, E. M. (2015). Inserting phase change lines into Microsoft Excel ${ }^{\circledR}$ graphs. Behavior Analysis in Practice, 8(2), 207-211. https://doi.org/10.1007/s40617-015-0078-8.

Fuller, T. C., \& Dubuque, E. M. (2018). Integrating phase change lines and labels into graphs in Microsoft Excel®. Behavior Analysis in Practice, 12(1), 293-299. https://doi.org/10.1007/s40617-0180248-6.

Google LLC. (2020). G Suite updates: New chart axis customization in Google Sheets: Tick marks, tick spacing, and axis lines. https:// gsuiteupdates.googleblog.com/2020/06/chart-axis-customizationgoogle-sheets-tick-marks-lines.html

Kubina, R. M., Kostewicz, D. E., Brennan, K. M., \& King, S. A. (2017). A critical review of line graphs in behavior analytic journals. Educational Psychology Review, 29(3), 583-598. https://doi.org/ 10.1007/s10648-015-9339-x.

Mattson, S. L., Higbee, T. S., Aguilar, J., Nichols, B., Campbell, V. E., Nix, L. D., Reinart, K. S., Peck, S., \& Lewis, K. (2020). Creating and sharing digital $\mathrm{ABA}$ instructional activities: A practical tutorial. Behavior Analysis in Practice, 13, 772-798. https://doi.org/10. 1007/s40617-020-00440-z.

Pritchard, J. K. (2008). A decade later: Creating single-subject design graphs with Microsoft Excel 2007TM. Behavior Analyst Today, 9(3-4), 153-161. https://doi.org/10.1037/h0100655.

Vanselow, N. R., \& Bourret, J. C. (2012). Online interactive tutorials for creating graphs with Excel 2007 or 2010. Behavior Analysis in Practice, 5(1), 40-46. https://doi.org/10.1007/BF03391816.

Publisher's Note Springer Nature remains neutral with regard to jurisdictional claims in published maps and institutional affiliations. 